\title{
Interesting case of upper GI bleed
}

Keywords: chronic pancreatitis, hemosuccus pancreaticus, endoscopy, contrast enhanced ct, embolization, surgery

\section{Introduction}

Upper GI bleeding is a common medical emergency with significant in hospital mortality if the treatment is delayed. We present a unique case of upper GI bleeding in a patient who frequently presented with malena to the hospital.

\section{Case report}

45 year old was sent to MAU with anaemia by his GP. His Haemoglobin was 39 with MCV 70 \& WBC 2.6. He visited his GP surgery due to fatigue, breathlessness \& one week history of malena. His past medical history included episode of pancreatitis 3 yrs ago. He drank 2 pints of beer/day. He was initially transfused and OGD \& Colonoscopy arranged which were both normal. Ultrasound abdomen showed splenomegaly. He subsequently underwent a CT scan which showed $2.2 \times 2.1 \mathrm{~cm}$ pseudo-aneurysm of the left gastric artery in close proximity to pancreas and lesser curve of stomach which was embolized. A further OGD \& Capsule endoscopy were done which were both normal. From November 2011 to July 2012 he had 3 further admissions with the same symptoms and underwent 3 OGD's, a colonoscopy, repeat capsule endoscopy and two CT angiograms which showed no residual pseudo-aneurysm.

In Aug 2012 he represented and a repeat CT scan showed recurrence of pseudo-aneurysm close to the lesser curve of stomach and distal body/tail of pancreas. Angiogram showed that the pseudoaneurysm had repurfused despite previous coils in left gastric artery and the left gastric artery was then again satisfactorily re-occluded (Figure 1).

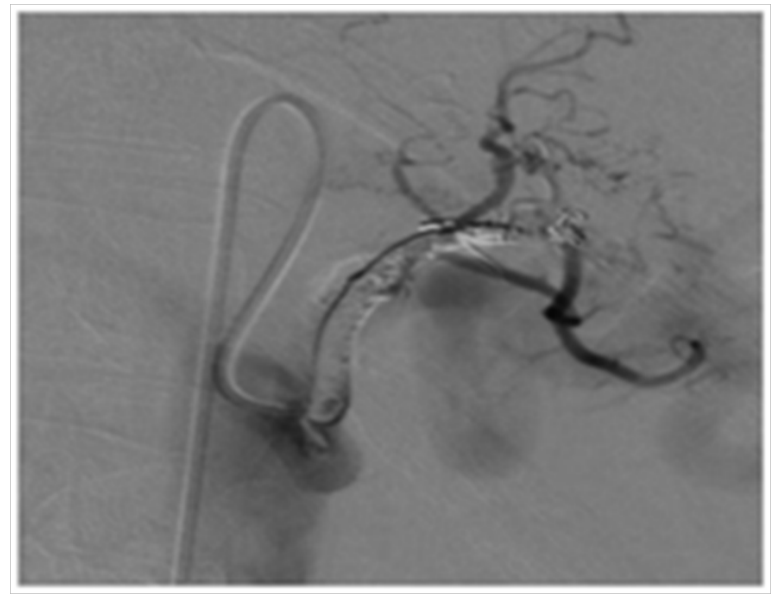

Figure I CT guided embolization of the left gastric artery.

In October 2012 he was admitted again with malena \& low haemoglobin. OGD was normal and CT angiogram showed no residual pseudo-aneurysm. RBC scan showed bleeding from region of duodenum/jejunum. Single balloon enteroscopy was performed which showed clot in second part of duodenum and blood oozing from the ampulla Figure 2 (A\&B). MRCP confirmed beaded dilation of pancreatic duct with normal biliary tree \& liver. Since CT had shown
Volume 4 Issue 6 - 2016

\author{
S Kadir,' M Jokhio, ${ }^{2}$ M Kelly ${ }^{3}$ \\ 'Royal Blackburn Hospital, UK \\ 2Macclesfield General Hospital, UK \\ ${ }^{3}$ University Hospital South Manchester, UK
}

Correspondence: Shanil Kadir, Consultant Gastroenterologist, Department of Gastroenterology, Royal Blackburn Hospital, Haslingden Road, Blackburn, BB2 3HH, UK, Tel 7950504753, Email drsks@email.com

Received: January 09, 2016 | Published: May 12, 2016

some splenic varices with splenomegaly a liver biopsy was conducted which showed no evidence of cirrhosis and the varices were thought to be secondary to splenic vein thrombosis. Subsequently a repeat $\mathrm{CT}$ angiogram did not show any recurrence of pseudo-aneurysm. His LFT's were always normal and so was his biliary anatomy. A diagnosis of Hemosuccus pancreaticus was made and his was referred to the regional hepato-biliary centre.
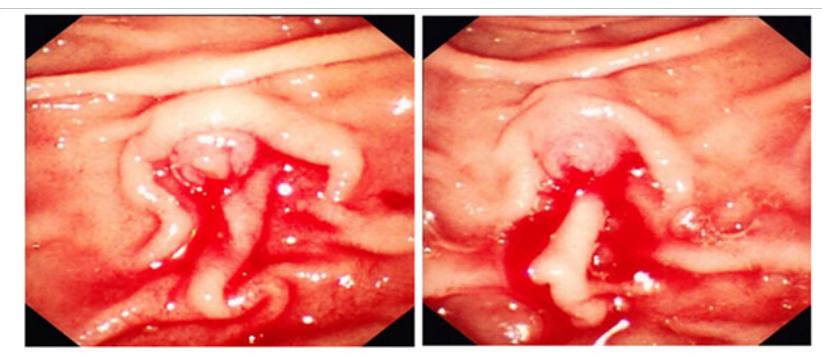

b.

Figure 2A \& B Endoscopic view of the ampulla of vater showing blood oozing from the ampulla with no periampullary ulcer.

\section{Discussion}

Hemosuccus pancreaticus is a rare and potentially life threatening cause of upper GI bleeding. It was first reported in 1931 by Lower \& Farrell who reported bleeding from aneurysm of splenic artery. ${ }^{1}$ Men aged 50-60 and patients with a history of alcohol induced chronic pancreatitis are most at risk. ${ }^{2}$ It is caused due to a rupture of splenic artery pseudo aneurysm communicating to the pancreatic duct or a communication between the peripancreatic artery and pancreatic pseudocyst. In $60-65 \%$ cases the culprit is splenic artery followed by gastro-duodenal, pancreato-duodenal, hepatic \& left gastric artery. Continuous thinning \& auto digestion of vessel wall by pancreatic enzyme along with cyst induced pressure necrosis causes development and subsequent rupture of pseudoaneursyms. ${ }^{3}$ 
It can present with epigastric pain radiating to the back in patients with history of chronic pancreatitis or with intermittent malena or hematemesis. Diagnosis is difficult to establish due to intermittent nature of bleeding which is most often not severe enough to cause hemodynamic instability. Endoscopy is the first line of investigation however Ultrasound and contrast enhanced CT can pick up pseudo cysts \& aneurysms and ERCP may help in establishing diagnosis of chronic pancreatitis or showing communicating pseudo cyst if other modalities fail to show anything. ${ }^{4}$ Some studies have reported Doppler ultrasound or dynamic ultrasound as diagnostic $\mathrm{c}^{5,6}$ and others have shown a $90 \%$ diagnostic rate using contrast enhanced CT. ${ }^{7}$ With endoscopy, bleeding can only be picked up only in 30\% of the cases even if you are using side viewing duodenoscope. ${ }^{8}$ Diagnosis can only be achieved by clinical suspicion, endoscopic, radiological and angiographic findings but even then in $52.9 \%$ cases no definite diagnosis is achieved especially in patients with fistula between pancreatic duct and aneurysm of peri-pancreatic vessels. ${ }^{7}$ Clots in the duodenum are thought to be indirect sign of Hemosuccus pancreaticus. ${ }^{2}$ Angiography is diagnostic reference standard with sensitivity of $96 \%{ }^{7}$

Management is with embolization of the culprit artery with surgery only in cases where embolization has failed, is not feasible or haemorrhage is uncontrolled. Surgery can also be considered in patients who have other indications of surgery i.e. pseudo cyst, pancreatic abscess, gastric outlet obstruction, obstructive jaundice or incapacitating pain. Most surgical series document a success rate of 70$85 \%$ with mortality rate of $20-25 \%{ }^{7,9,10}$ After endovascular treatment some studies have shown that the patient is free of symptoms ${ }^{11,12}$ with others showing a recurrence rate of $30 \% .{ }^{9}, 13$

\section{Learning points}

Hemosuccus pancreaticus should always be thought of in GI bleed patients with background of chronic pancreatitis or pancreatic pseudo cyst where no other cause of bleeding has been demonstrated. Diagnosis is not straight forward and different diagnostic modalities along with high clinical suspicion may be required to demonstrate Hemosuccus pancreaticus. Mortality is between $12-57 \%$ in treated and $90-100 \%$ in untreated ruptures. ${ }^{14}$

\section{Acknowledgments}

None.

\section{Conflicts of interset}

The author declares there is no conflict of interest.

\section{References}

1. Toyoki Y, Hakamada K, Narumi S, et al. Hemosuccus pancreaticus: Problems and pitfalls in diagnosis and treatment. World J Gastroenterol. 2018;14(17):2776-2779.

2. Etienne S, Pessaux P, Tuech JJ, et al. Hemosuccus pancreaticus: a rare cause of gastrointestinal bleeding. Gastroenterol Clin Biol. 2005;29(3):237-242.

3. Sugiki $T$, Hatori $T$, Imaizumi $T$, et al. Two cases of hemosuccus pancreaticus in which hemostasis was achieved by transcatherter arterial embolization. J Hepatobiliary Pancreat Surg. 2003;10(6):450-454.

4. Sakorafas GH, Sarr MG, Farley DR, et al. Hemosuccus pancreaticus complicating chronic pancreatitis:an obscure cause of upper GI bleeding. Langenbeck's Arch Surg. 2000;385(2):124-128.

5. Risti B, Marincek B, Jost R, et al. Haemosuccus pancreaticus as a source of obscure upper gastrointestinal bleeding: three cases and literature review. Am J Gastroenterol. 1995;90(10):1878-1880.

6. Benz CA, Jakob P, Jakobs R, et al. Hemosuccus pancreaticus--a rare cause of gastrointestinal bleeding: diagnosis and interventional radiological therapy. Endoscopy. 2000;32(5):428-431.

7. Vimalraj V, Kannan DG, Sukumar R, et al. Haemosuccus pancreaticus: diagnostic and therapeutic challenges. HPB (Oxford). 2009;11(4):345350 .

8. Koren M, Kinova S, Bedeova J, et al. Hemosuccus pancreaticus. Bratisl Lek Listy. 2008;109(1):37-41.

9. de Perrot M, Berney T, Bühler L, et al. Management of bleeding pseudoaneurysms in patients with pancreatitis. Br J Surg. 1999;86(1):2932.

10. Bergert H, Hinterseher I, Kersting S, et al. Management and outcome of hemorrhage due to arterial pseudoaneurysms in pancreatitis. Surgery. 2005;137(3):323-328.

11. Gambiez LP, Ernst OJ, Merlier OA, et al. Arterial embolization for bleeding pseudocysts complicating chronic pancreatitis. Arch Surg. 1997;132(9):1016-1021.

12. Huizinga WK, Kalideen JM, Bryer JV, et al. Control of major haemorrhage associated with pancreatic pseudocysts by transcatheter arterial embolization. Br J Surg. 1984;71(2):133-136.

13. Boudghène F, L Herminé C, Bigot JM. Arterial complications of pancreatitis: diagnostic and therapeutic aspects in 104 cases. $J$ Vasc Interv Radiol. 1993;4(4):551-558.

14. Kumar B, Jha S. Hemosuccus pancreaticus due to rupture of a gastroduodenal artery pseudoaneurysm. Hosp Physician. 2007;43:6164.

\section{Funding}

None. 